\title{
Ensino complementar de micologia médica na modalidade a distância em meio à pandemia da COVID-19: um relato de experiência
}

\author{
Complementary distance education in medical mycology in COVID-19 pandemic times: an \\ experience report
}

Educación complementaria a distancia de micología médica en medio de la pandemia de COVID19: un relato de experiencia

\section{Resumo}

O presente estudo objetiva relatar a experiência na aplicação de um curso de extensão, o qual foi proposto como complemento de ensino na área da micologia médica para estudantes de cursos de graduação na área da saúde. Devido à pandemia do novo coronavírus, o curso foi oferecido na modalidade de ensino a distância. $\mathrm{O}$ curso abordou desde as características gerais dos fungos até a identificação de organismos clinicamente significativos nas infecções fúngicas, destacando as micoses superficiais, cutâneas, subcutâneas, sistêmicas, invasivas e as micotoxinas. As principais estratégias de aprendizagem foram as videoaulas gravadas, as atividades assíncronas disponibilizadas para complementar o aprendizado e o estudo de casos clínicos em grupos virtuais nas atividades síncronas. Com relação ao aprendizado dos estudantes, foi possível observar uma maior familiaridade dos participantes com relação aos organismos causadores das infecções fúngicas, principalmente, pela assertividade na resolução dos casos clínicos. Em relação às estratégias metodológicas, verificou-se que foram adequadas, com destaque à necessidade de aumentar o número de encontros síncronos e o tempo de estudo devido à densidade dos conteúdos. Este curso de extensão oferecido como um complemento para o ensino de micologia mostrou ser uma experiência positiva para aumentar a efetividade do aprendizado nesta área do conhecimento.

Palavras-chave: Micologia; Educação a distância; Educação médica continuada.

\begin{abstract}
The current study aims to report the experience in the application of an extension course, which was proposed as a complementary education in medical mycology for undergraduate students in the health area. Due to the new coronavirus pandemic, the course was offered in the distance education modality. The course covered about the general characteristics of fungi and the identification of significant organisms in fungal infections, highlighting superficial, cutaneous, subcutaneous, systemic, invasive mycoses and mycotoxins. The main learning strategies were recorded video classes, asynchronous activities available to complement the learning and clinical case studies in
\end{abstract}


virtual groups in synchronous activities. Regarding the students learning, it was possible to observe a greater familiarity of the participants in relation to the organisms causing fungal infections, mainly due to the assertiveness in the resolution of clinical cases. Regarding the methodological strategies, they were adequate, however, could be need to increase the number of synchronous activities and the study time due to the density of the contents. This extension course offered as a complement distance education in medical mycology showed to be a positive experience to increase the effectiveness of learning in this area of knowledge.

Keywords: Mycology; Distance education; Continuing medical education.

\section{Resumen}

El presente estudio tiene como objetivo reportar la experiencia en la aplicación de un curso de extensión, el cual fue propuesto como complemento a la docencia en el campo de la micología médica para estudiantes de pregrado en el campo de la salud. Debido a la nueva pandemia de coronavirus, el curso se ofreció en la modalidad de educación a distancia. El curso abarcó desde las características generales de los hongos hasta la identificación de organismos clínicamente significativos en las infecciones fúngicas, destacando las micosis superficiales, cutáneas, subcutáneas, sistémicas, invasivas y micotoxinas. Las principales estrategias de aprendizaje fueron las lecciones en video grabadas, las actividades asincrónicas puestas a disposición para complementar el aprendizaje y los estudios de casos clínicos en grupos virtuales en las actividades sincrónicas. Cuanto, al aprendizaje de los estudiantes, se pudo observar una mayor familiaridad de los participantes con relación a los organismos causantes de infecciones fúngicas, principalmente debido al asertividad en la resolución de casos clínicos. Cuanto, a las estrategias metodológicas, se encontró que fueron adecuadas con énfasis en la necesidad de incrementar el número de reuniones sincronizadas y el tiempo de estudio debido a la densidad de los contenidos. Este curso de extensión ofrecido como complemento a la educación de la micología resultó ser una experiencia positiva para incrementar la efectividad del aprendizaje en esta área del conocimiento.

Palabras clave: Micología; Educación a distancia; Educación médica continua.

\section{Introdução}

Infecções fúngicas são comumente encontradas na população, afetando mais de 1 bilhão de pessoas ao redor do mundo (Bongomin et al., 2017) e se expandindo a medida que o número de indivíduos vulneráveis (transplantados, pacientes imunocomprometidos, nascidos prematuros e idosos) aumenta (Vallabhaneni et al., 2016). As estimativas globais sugerem que mais de 1,5 milhão de pessoas morrem de infecções fúngicas, 10 milhões sofrem de alergia fúngica grave e 100 milhões de mulheres apresentam recorrentes infecções vulvovaginais anualmente (Brown et al., 2012). No Brasil, a incidência de infecções fúngicas oportunistas como a candidemia é de 3 a 15 vezes maior que a reportada nos países da Europa e América do Norte (Nucci et al., 2010), e, na América Latina, é o país que mais sofre de micoses endêmicas (Colombo et al., 2011).

Se, por um lado, o número de pacientes imunocomprometidos acometidos por infecções fúngicas continua a aumentar, por outro lado, a formação educacional de médicos micologistas ainda é deficitária (Steinbach et al., 2003). Devido à ubiquidade e variedade de patógenos fúngicos existentes, a área da micologia médica apresenta um desafio diagnóstico e terapêutico para profissionais da saúde. A capacidade de disseminação e invasão em tecidos e sistemas, assim como a reação do hospedeiro às infecções, pode levar a um diagnóstico tardio e um tratamento inadequado. Desta forma, a educação continuada e o treinamento apropriado podem desenvolver competências diagnósticas importantes para melhorar a agilidade no tratamento de pacientes com infecções fúngicas invasivas (Rosner et al., 2002).

Neste contexto, é imprescindível que haja estudo contínuo da micologia médica pelos profissionais da área da saúde, considerando que, quanto mais precoce for realizado o diagnóstico e o tratamento da infecção, melhor será o desfecho do quadro clínico. Somado-se a isso, a pandemia do novo coronavírus (Sars-CoV-2), desde o início de 2020, levou a população a aderir ao isolamento social (Sohrabi et al., 2020). Sabe-se que, ao longo da história da humanidade, pandemias moldaram profundamente a sociedade, impactando os métodos e contextos educacionais. Do mesmo modo, a pandemia do COVID-19 é vista por diversos autores como o estopim para a revolução da educação a distância e a educação domiciliar (homeschooling) (Spielman \& Sunavala-Dossabhoy, 2021). Diante dessa situação emergencial, a qual trouxe como consequência o distanciamento social, a produção de conteúdos online tornou-se uma das principais opções para garantir a continuidade da educação através das diferentes metodologias de ensino. 
Diante deste cenário, como ensino complementar à área de micologia médica, o curso de extensão intitulado "Infecções fúngicas e micotoxinas: desafios para profissionais da área médica" foi proposto aos estudantes dos cursos de graduação na área da saúde da Universidade Federal de Santa Catarina (UFSC). O objetivo do curso consistiu em apresentar aos estudantes os desafios relacionados à identificação de infecções fúngicas, e, com isso, torná-los familiarizados com os aspectos patogênicos e epidemiológicos da infecção, no intuito de possibilitar um diagnóstico adequado e uma terapia mais rápida e eficaz.

O curso de extensão foi oferecido totalmente na modalidade de ensino a distância, seguindo a tendência mundial da conversão de diferentes modalidades de ensino presencial em digital (Afghani, 2021; Dorfsman \& Horenczyk, 2021). Para adaptação do curso nessa modalidade, tornou-se necessária a implementação de estratégias e técnicas de ensino que permitissem manter o conteúdo atrativo e interativo, e que promovessem o engajamento dos estudantes na realização de atividades colaborativas (Babacan et al., 2021).

Desta forma, o presente estudo objetiva relatar a experiência na aplicação do curso de extensão intitulado "Infecções fúngicas e micotoxinas: desafios para profissionais da área médica", oferecido na modalidade à distância, o qual foi proposto como complemento de ensino na área da micologia médica para estudantes de cursos de graduação na área da saúde.

\section{Metodologia}

\subsection{Desenho do estudo}

Trata-se de uma pesquisa com abordagem qualitativa, exploratória, descrita no formato de relato de experiência (Gil, 2017).

\subsection{Público-alvo}

O curso de extensão foi destinado a acadêmicos de Medicina da UFSC campus Araranguá, bem como a estudantes de outros cursos da área da saúde do campus de Florianópolis. A divulgação ocorreu para todos os cursos, não havendo prérequisito na formação para realizar a inscrição. Entretanto, o número de vagas foi limitado $(n=24)$ devido ao curso ser oferecido pela primeira vez na modalidade a distância e para que o encontro síncrono previsto tivesse menos estudantes, com o intuito de permitir que todos participassem dos casos clínicos discutidos. Desta forma, também seria possível fornecer aos estudantes um parecer de cada atividade realizada durante o curso, de forma individual.

\subsection{Local e período de realização da pesquisa}

O curso de extensão "Infecções fúngicas e micotoxinas: desafios para profissionais da área médica", depois de aprovado pela UFSC, foi organizado na plataforma Moodle (Modular Object Oriented Distance Learning), e ministrado na modalidade a distância entre agosto e novembro de 2020.

\subsection{Planejamento inicial do curso de extensão}

Os estudantes de graduação na área da saúde da UFSC - Campus Araranguá foram consultados quanto ao interesse em participar de um curso de extensão que tinha como foco infecções fúngicas e micotoxinas. Para isto, foi disponibilizado aos estudantes, via redes sociais, uma enquete informal contendo questionamentos que norteariam a construção do curso.

\subsection{Estruturação do curso de extensão}

A organização do curso se deu conforme as respostas dos estudantes sobre os questionamentos disponibilizados inicialmente via redes sociais, considerando, principalmente, a carga horária total do curso e a modalidade de apresentação. 
O curso de extensão foi dividido em quatro módulos com uma carga horária total de 16 horas, compreendendo atividades assíncronas e síncronas. As atividades assíncronas não necessitaram da presença do tutor do curso e, portanto, foram realizadas exclusivamente pelo aluno no horário e forma que acharam conveniente. Já as atividades síncronas, necessitaram da presença, num ambiente on-line, tanto dos alunos como do tutor, onde os encontros ocorreram em horários e dias previamente definidos, conforme disponibilidade dos envolvidos. Por isso, o encontro síncrono foi realizado pela plataforma Google Meet, sendo restrito apenas aos participantes do curso e do tutor.

Os módulos foram disponibilizados a cada 7 dias, e seus temas estão expostos na Tabela 1.

Tabela 1. Módulos do curso e os temas abordados.

\begin{tabular}{ll}
\hline MÓDULOS & TEMAS \\
\hline Módulo I & Características gerais dos fungos \\
Módulo II & Micoses superficiais, cutâneas e subcutâneas \\
Módulo III & $\begin{array}{l}\text { Micoses sistêmicas causadas por fungos dimórficos e micoses invasivas causadas por fungos } \\
\text { oportunistas }\end{array}$ \\
Módulo IV & Micotoxinas \\
\hline
\end{tabular}

Fonte: Autores (2022).

$\mathrm{Na}$ explanação dos temas em cada módulo, foram apresentadas a morfologia dos patógenos, diagnóstico laboratorial, epidemiologia das micoses, manifestações clínicas dos pacientes e agentes antifúngicos (Brooks et al., 2012; Tavares 2013; Murray et al., 2017). Como estudo complementar para o curso de extensão, a principal obra indicada aos estudantes foi o trabalho de Murray et al. (2017).

As principais estratégias de aprendizagem foram: (a) utilização de videoaulas pré-gravadas com aulas expositivas adaptadas para o ambiente virtual (ABED, 2018); (b) uso de imagens para facilitar a visualização dos temas abordados em cada videoaula gravada; (c) atividades assíncronas disponibilizadas para complementar o aprendizado, em formato de questionário, resolução de casos clínicos e discussão de relatos de casos e experiências sobre o assunto discutido (Brito, 2010); (d) estudo e discussão de casos clínicos em grupos virtuais nas atividades síncronas (Peixoto et al., 2018) e (e) fórum de dúvidas para discussão sobre cada módulo apresentado. As atividades postadas pelos participantes foram avaliadas pelo tutor, as quais também foram validadas como presença e participação no curso.

\section{Resultados e Discussão}

No total, houve 106 inscritos para o curso de extensão intitulado "Infecções fúngicas e micotoxinas: desafios para profissionais da área médica". No entanto, devido ao número limitado de vagas, um total de 24 estudantes participaram desse curso de extensão. As vagas foram preenchidas por ordem de inscrição. Do total, 23 eram do curso de Medicina e apenas 1 do curso de Ciências Biológicas.

A pandemia gerada pelo COVID-19 trouxe novas necessidades em relação a utilização de ferramentas e estratégias educacionais (Spielman \& Sunavala-Dossabhoy, 2021). A aplicação deste curso ocorreu nesta fase de isolamento social, possibilitando levar o conhecimento sobre o tema para estudantes da área da saúde de uma forma totalmente à distância. Desta forma, foi possível efetuar um complemento aos estudos de micologia médica nesse momento de pandemia.

De acordo com o levantamento já realizado por Steinbach et al. (2003), apesar do número de fungos oportunistas ser crescente, os diferentes tipos de tratamento também aumentaram de forma significativa. Neste caso, a exigência de maior profundidade do conhecimento em micologia médica deve ser incentivada. O conhecimento na área pode melhorar um diagnóstico e a escolha terapêutica diante de uma infecção suspeita. 
Neste sentido, ao final deste curso de extensão, esperou-se familiarização dos estudantes com os organismos clinicamente significativos nas infecções fúngicas e que eles compreendessem a importância de um diagnóstico assertivo das micoses para o uso apropriado de agentes terapêuticos. Para atingir estes resultados, o curso foi organizado em quatro módulos, cada qual disponibilizado com os temas escolhidos do curso de extensão.

A Figura 1 apresenta o fluxograma do trabalho apresentando resumidamente todos os módulos do curso de extensão, bem como as metodologias empregadas.

Figura 1. Fluxograma do trabalho contendo os módulos apresentados no curso de extensão oferecido na modalidade a distância.

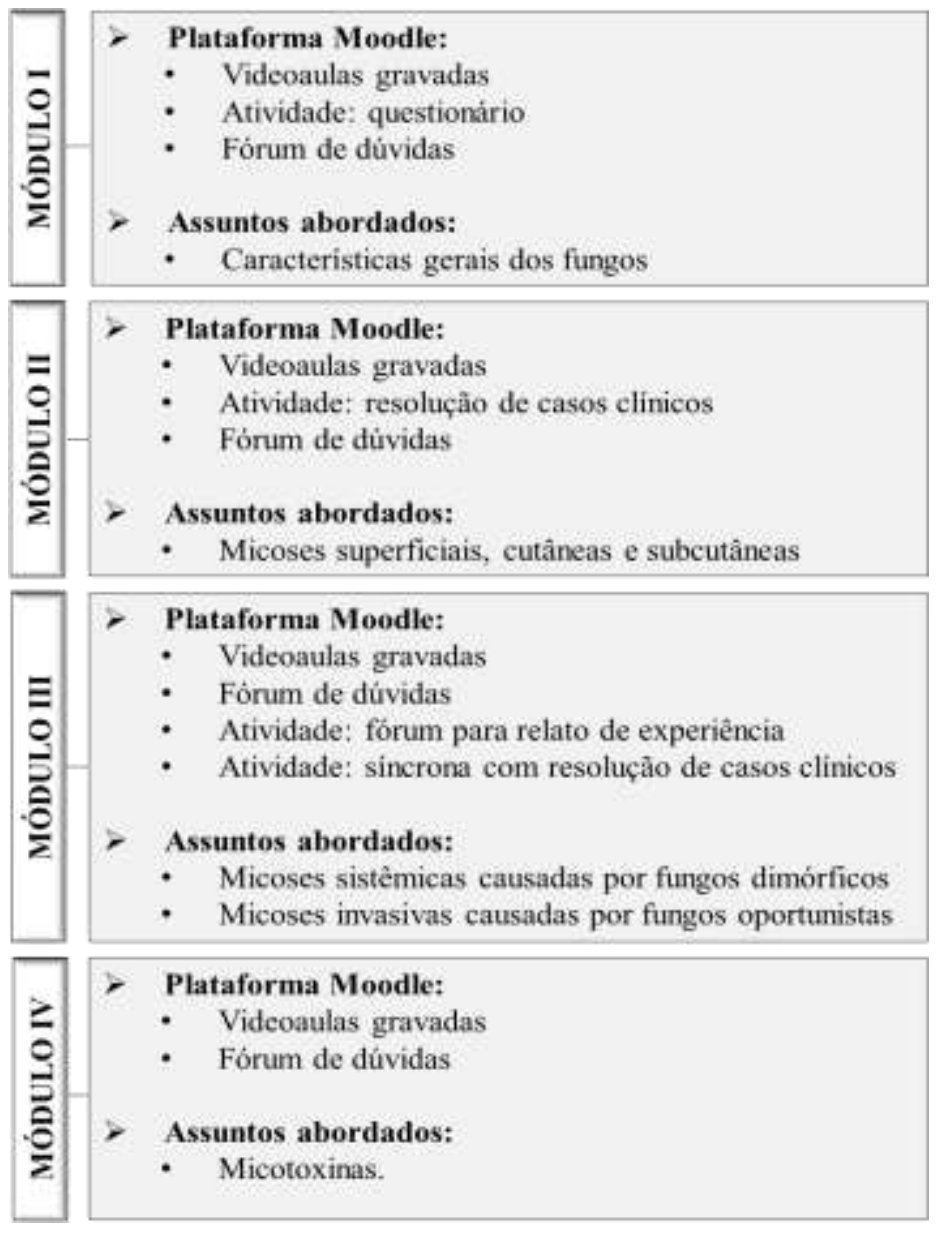

Fonte: Autores (2022).

Em todos os módulos a exposição de conteúdos ocorreu principalmente por meio de videoaulas pré-gravadas, portanto, de modo assíncrono, permitindo que o aluno fizesse seu estudo no horário e ambiente que desejasse. Isto constitui um facilitador, pois o ambiente acadêmico dos cursos de graduação em Saúde, especialmente o de Medicina, é quase sempre muito estressor ao aluno, pois carrega uma rotina intensa de atividades (Kam et al., 2019). O curso de extensão seguiu a produção de videoaulas mantendo a expressividade e a oralidade do tutor durante exposição do conteúdo. Ao mesmo tempo, os slides tiveram elementos de imagem que pudessem trazer informações aos estudantes de forma clara e objetiva, sendo a fala do tutor alinhada com os registros dos slides (Penteado \& Costa, 2021).

O primeiro módulo foi intitulado como Introdução ao Curso de Extensão. Este módulo foi totalmente assíncrono, no qual foram disponibilizadas duas videoaulas e uma atividade. Na primeira videoaula foi realizada uma apresentação do curso de extensão com informações da ministrante, do funcionamento do curso na modalidade a distância e do conteúdo 
programático. Na segunda videoaula foi apresentada uma visão geral sobre as características dos fungos incluindo classificação, estrutura e reprodução. Para complementar os estudos deste módulo, foi elaborada uma atividade em formato de questionário com perguntas sobre o tema, apresentando alternativas com apenas uma resposta correta. A correção desta atividade foi realizada automaticamente pela plataforma Moodle e os estudantes tiveram o conhecimento da resposta correta contendo o feedback logo após a finalização do questionário. Se caso houvesse alguma resposta incorreta após feedback, era possível realizar uma nova tentativa para responder o questionário.

O segundo módulo também foi totalmente assíncrono, no qual foram disponibilizadas videoaulas gravadas abordando os organismos clinicamente significativos nas infecções fúngicas, com destaque às micoses superficiais (camadas mais externas da pele e dos pelos) cutâneas (camadas mais profundas da epiderme, pelos e unhas) e subcutâneas (derme, tecidos subcutâneos, músculo e tecido conjuntivo). Após as videoaulas, os estudantes realizaram uma atividade de resolução de dois casos clínicos. Após o envio da atividade por todos os estudantes, a ministrante apresentou um feedback para a turma, com os comentários sobre os pontos de discussão. Os estudantes receberam as correções destes casos clínicos pelo e-mail cadastrado na plataforma Moodle. É importante destacar que todos os estudantes conseguiram alcançar a hipótese diagnóstica dos casos clínicos corretamente.

O terceiro módulo foi dividido em atividades assíncronas e síncronas. Na atividade assíncrona foi disponibilizada uma videoaula com a apresentação dos fungos dimórficos como patógenos das micoses sistêmicas e, também, as micoses invasivas causadas por fungos oportunistas. Já a atividade síncrona foi elaborada para a discussão de cinco casos clínicos com duração de aproximadamente duas horas na presença virtual dos estudantes, através de videoconferência. Os casos clínicos foram enviados previamente neste módulo, juntamente com algumas perguntas, como pontos de discussão para que os estudantes pudessem resolvê-los antes da reunião virtual. O objetivo desta atividade foi de discutir os casos clínicos em conjunto, propiciando a troca de ideias, sugestões e resolução de dúvidas. Um modelo de caso clínico discutido na atividade síncrona está apresentado no Quadro 1, com a adição de algumas imagens relacionadas aos sintomas e a identificação do agente etiológico (não apresentadas).

Quadro 1. Modelo de um dos casos clínicos discutidos na atividade síncrona.

\begin{tabular}{|c|c|}
\hline MODELO DE CASO CLÍNICO & PONTOS DE DISCUSSÃO \\
\hline $\begin{array}{l}\text { Paciente do sexo feminino, } 35 \text { anos, procedente do interior do } \\
\text { estado de Santa Catarina, sem comorbidade prognóstica, chega } \\
\text { ao pronto socorro com quadro de cefaleia há } 15 \text { dias com forte } \\
\text { intensidade, refratária ao uso de analgésicos, associada a astenia, } \\
\text { adinamia, perda de peso, náuseas e vômitos. Os sintomas } \\
\text { evoluíram com piora progressiva do quadro. A paciente } \\
\text { apresentou paralisia do nervo craniano VI à esquerda. A } \\
\text { tomografia computadorizada do crânio mostrou lesão expansiva } \\
\text { em região frontal direita e occipital esquerda exercendo efeito de } \\
\text { massa. Apresentou sorologias para sífilis, hepatites A, B e C, } \\
\text { citomegalovírus, toxoplasmose e HIV todas negativas. A pressão } \\
\text { liquórica estava aumentada e o líquor apresentava leucocitose } \\
\text { leve a moderada com diminuição dos níveis de glicose e } \\
\text { elevação de proteinorraquia. Mantendo o quadro clínico e } \\
\text { papiledema bilateral, a paciente foi submetida à ressecção } \\
\text { microcirúrgica para exérese da lesão frontal direita em caráter de } \\
\text { urgência. O exame anatomopatológico evidenciou crescimento } \\
\text { de leveduras capsuladas abundantes (Adaptado de Júnior et al. } \\
\text { 2015). }\end{array}$ & $\begin{array}{l}\text { 1. Qual a principal hipótese diagnóstica? } \\
\text { 2. Existe fatores de riscos para esta infecção? } \\
\text { 3. Qual o principal agente etiológico envolvido nesta infecção? } \\
\text { 4. Como o paciente pode ter adquirido a infecção por este agente } \\
\text { etiológico? } \\
\text { 5. Quais testes poderiam ser adicionados na avaliação } \\
\text { micológica para confirmação da espécie fúngica? } \\
\text { 6. Qual a sugestão de tratamento? } \\
\text { 7. Quais as possíveis complicações se o paciente não receber o } \\
\text { tratamento correto? }\end{array}$ \\
\hline
\end{tabular}

Fonte: Autores (2022).

O uso de estudos de caso na educação médica já é bem estabelecido, sendo considerado preferido a outros métodos inovadores como o ABP (Aprendizado Baseado em Problemas, do inglês, Problem Based Learning) (Srinivasan et al., 2007). Além disso, se constitui como base para o desenvolvimento do raciocínio clínico, tão importante para elaboração do 
diagnóstico correto e, a partir deste, definir a melhor conduta a ser tomada por um profissional da saúde (Peixoto et al., 2018). Nesse sentido, algumas estratégias vêm sendo usadas para difundir este método no ambiente à distância utilizando o Moodle (Mezzari et al., 2012) ou mesmo salas virtuais para discussão (Abensur et al., 2007).

A atividade síncrona incentivou a participação ativa dos estudantes no processo de ensino-aprendizagem, discutindo em conjunto os questionamentos levantados. Além disso, auxiliou na avaliação das competências dos estudantes relacionadas à cognição e à comunicação (Arafeh, 2011), o pensamento crítico, a reflexão e a aprendizagem partindo da experiência e a argumentação (Bosque, 2012). Também proporcionou feedback rápido aos estudantes que foram incentivados a responder os questionamentos dos casos, atendendo à necessidade imediata de dúvidas que surgiram durante a discussão (Gallo, 2011).

Além das atividades relacionadas a resolução dos casos clínicos, também é interessante ressaltar a atividade do módulo III, em que uma das opções para participação do fórum era relatar uma experiência sobre alguma micose que estava sendo estudada no módulo atual ou nos módulos anteriores. A experiência poderia ser do próprio estudante ou de outra pessoa em que o caso fosse conhecido. Neste fórum, a atividade pretendeu relatar a micose e descrever resumidamente sobre ela. Foi possível perceber o quanto os estudantes conseguirem explicar com maiores detalhes o seu relato de experiência trazendo informações importantes que foram obtidas principalmente através das videoaulas disponibilizadas.

Por fim, no quarto módulo foi apresentada uma videoaula sobre o tema micotoxinas (toxinas produzidas por fungos filamentosos). A maioria dos estudantes da área da saúde aprende muito pouco sobre micotoxinas, embora seus efeitos tóxicos sobre os seres humanos sejam bem reconhecidos (Altomare et al., 2021; Haque et al., 2020). Portanto, esse módulo foi introduzido ao curso para enfatizar sobre a importância das toxinas produzidas pelos fungos toxigênicos e seus malefícios para a saúde humana.

Em todos os módulos, a interação entre os estudantes e professor também foi possível pela plataforma Moodle usando os fóruns de discussão. Por meio desse recurso, os estudantes puderam participar de forma interativa e colaborativa e construir seu próprio conhecimento, baseado na teoria construtivista (Lima, 2021). Para cada módulo foi aberto um fórum de dúvidas onde os acadêmicos poderiam acrescentar um tópico de discussão sobre o tema disponibilizado no módulo. Na atividade assíncrona a presença deste fórum possibilita ao acadêmico tirar suas dúvidas durante o estudo do módulo. Sempre que surgiram, todas as dúvidas adicionadas pelos estudantes no fórum foram sanadas pelo ministrante o mais rápido possível. Desta forma, os estudantes puderam visualizar e inclusive questionar sobre a resposta, quando necessário.

A partir da aplicação inédita deste curso de extensão, foi possível observar aspectos positivos e os aspectos que precisam ser melhorados em uma próxima edição. Por meio da elaboração do curso na modalidade a distância, foi possível utilizar vários recursos e ferramentas que estão disponíveis, com o intuito de melhorar a exposição das aulas, como as videoaulas e as atividades síncronas e assíncronas. Foi possível observar que os estudantes ficaram satisfeitos com este curso de extensão oferecido na modalidade a distância e demonstraram interesse em realizar outros cursos neste mesmo formato.

\section{Considerações Finais}

Com relação ao aprendizado dos acadêmicos, o curso de extensão atingiu os objetivos propostos uma vez que tanto nas atividades assíncronas quanto nas síncronas foi possível observar uma maior familiaridade com os temas estudados e assertividade na resolução dos casos clínicos.

Quanto aos aspectos que podem ser melhorados, o tempo de duração do curso poderia ser maior devido ao extenso conteúdo disponibilizado por meio das videoaulas. Além disso, a atividade síncrona poderia ser realizada mais vezes durante o curso, uma vez que a participação dos acadêmicos foi muito produtiva. Destacou-se, ainda, o interesse dos participantes pelos estudos de casos clínicos, os quais aproximam a teoria de contextos reais e aplicados.

Como limitações, destaca-se o reduzido número de vagas disponibilizadas na primeira versão, sendo possível oferecer 
mais vagas, já que o curso é realizado totalmente à distância. Em trabalhos futuros sugere-se avaliar formalmente (com uso questionário on-line) o nível de satisfação dos participantes deste curso, verificando suas percepções em relação ao aprendizado do conteúdo, adequação das metodologias à distância e demais ferramentas aplicadas.

A utilização de recursos na plataforma Moodle auxiliaram na organização deste curso de extensão, oferecendo estratégias de ensino-aprendizagem na modalidade a distância. O curso de extensão, como uma forma de complemento para o ensino de micologia na graduação dos cursos da área da saúde, pode aumentar a efetividade do aprendizado nesta área do conhecimento e a formação profissional dos estudantes.

\section{Referências}

ABED. Associação Brasileira de Educação a Distância. (2018). Analytic report of distance learning in Brazil. InterSaberes.

Abensur, S. I., Abensur, H., Malheiros, D. M. A. C., \& Zatz, R. (2007). Uso da internet como um ambiente para discussão de casos clínicos. Revista Brasileira de Educação Médica, 31(3), 291-295. https://doi.org/10.1590/S0100-55022007000300012

Afghani, B. (2021). COVID-19 pandemic: a catalyst for transformation of a summer online research program. Medical Education Online, 26(1), 1886029. https://doi.org/10.1080/10872981.2021.1886029

Altomare, C., Logrieco, A. F., \& Gallo, A. (2021). Mycotoxins and Mycotoxigenic Fungi: Risk and Management. A Challenge for Future Global Food Safety and Security (Ó. Zaragoza \& A. B. T.-E. of M. Casadevall (eds.); pp. 64-93). Elsevier. https://doi.org/10.1016/B978-0-12-819990-9.00032-9

Arafeh, J. (2011). Simulation-Based Training The Future of Competency? The Journal of Perinatal \& Neonatal Nursing, 25, 171-174. https://doi.org/10.1097/JPN.0b013e3182116e55

Babacan, S., \& Dogru Yuvarlakbas, S. (2021). Digitalization in education during the COVID-19 pandemic: emergency distance anatomy education. Surgical and Radiologic Anatomy. https://doi.org/10.1007/s00276-021-02827-1

Barbeau, M. L., Johnson, M., Gibson, C., \& Rogers, K. A. (2013). The development and assessment of an online microscopic anatomy laboratory course. Anatomical Sciences Education, 6(4), 246-256. https://doi.org/https://doi.org/10.1002/ase.1347

Bongomin, F., Gago, S., Oladele, R. O., \& Denning, D. W. (2017). Global and Multi-National Prevalence of Fungal Diseases-Estimate Precision. Journal of Fungi (Basel, Switzerland), 3(4), 57. https://doi.org/10.3390/jof3040057

Bosque, E. (2012). Toward salience: an application of integrative, case-based, nursing education for neonatal advanced practice. Advances in Neonatal Care : Official Journal of the National Association of Neonatal Nurses, 12(5), 292-302. https://doi.org/10.1097/ANC.0b013e318262499b

Brito, J. A. (2010). Engajamento em atividades assíncronas na modalidade de ensino a distância: requisitos de interfaces colaborativas. Universidade Federal de Pernambuco.

Brooks, G.F., Carroll, K.C., Butel, J.S., Morse, S.A., Mietzner, T. A. (2012). Microbiologia médica de Jawetz, Melnick e Adelberg (25. ed).

Brown, G. D., Denning, D. W., Gow, N. A. R., Levitz, S. M., Netea, M. G., \& White, T. C. (2012). Hidden killers: human fungal infections. Science Translational Medicine, 4(165), 165rv13. https://doi.org/10.1126/scitranslmed.3004404

Castillo, J. (2000). The decline of clinical laboratory science programs in colleges and universities. Journal of Allied Health, $29,30-35$.

Colombo, A. L., Tobón, A., Restrepo, A., Queiroz-Telles, F., \& Nucci, M. (2011). Epidemiology of endemic systemic fungal infections in Latin America. Medical Mycology, 49(8), 785-798. https://doi.org/10.3109/13693786.2011.577821

Dorfsman, M., \& Horenczyk, G. (2021). The coping of academic staff with an extreme situation: The transition from conventional teaching to online teaching. Education and Information Technologies. https://doi.org/10.1007/s10639-021-10675-0

Gallo, A.-M. (2011). Beyond the classroom: using technology to meet the educational needs of multigenerational perinatal nurses. The Journal of Perinatal \& Neonatal Nursing, 25(2), 195-199. https://doi.org/10.1097/JPN.0b013e3182163993

Gil, A. C. (2017). Como elaborar projetos de pesquisa (5th ed.). Atlas.

Githang'a, D., Anzala, O., Mutegi, C., \& Agweyu, A. (2019). The effects of exposures to mycotoxins on immunity in children: A systematic review. Current Problems in Pediatric and Adolescent Health Care, 49(5), 109-116. https://doi.org/10.1016/j.cppeds.2019.04.004

Haque, M. A., Wang, Y., Shen, Z., Li, X., Saleemi, M. K., \& He, C. (2020). Mycotoxin contamination and control strategy in human, domestic animal and poultry: A review. Microbial Pathogenesis, 142, 104095. https://doi.org/https://doi.org/10.1016/j.micpath.2020.104095

Kam, S., Toledo, A., Pacheco, C., Souza, G., Santana, V., Bonfá-Araujo, B., \& Custodio, C. (2019). Estresse em Estudantes ao longo da Graduação Médica. Revista Brasileira de Educação Médica, 43, 246-253. https://doi.org/10.1590/1981-5271v43suplemento1-20180192

Lima, J. M. M. (2021). Plataforma Moodle: A educação por mediação tecnológica. Revista Científica Multidisciplinar Núcleo Do Conhecimento, 7(1), 17-37. https://www.nucleodoconhecimento.com.br/educacao/plataforma-moodle\%0D 
Research, Society and Development, v. 11, n. 1, e40611125237, 2022

(CC BY 4.0) | ISSN 2525-3409 | DOI: http://dx.doi.org/10.33448/rsd-v11i1.25237

Mezzari, A., Iser, I., Wiebbelling, A. M. P., \& Tarouco, L. (2012). O Uso do Moodle como Reforço ao Ensino Presencial de Parasitologia e Micologia no Curso de Graduação em Medicina. Revista Brasileira de Educação Médica, 36(4), 557-563.

Murray, P.R.; Rosenthal, K.S.; Pfaller, M. A. (2017). Microbiologia médica (Elsevier (ed.); (8a ed.).

Nucci, M., Queiroz-Telles, F., Tobón, A. M., Restrepo, A., \& Colombo, A. L. (2010). Epidemiology of Opportunistic Fungal Infections in Latin America Clinical Infectious Diseases, 51(5), 561-570. https://doi.org/10.1086/655683

Peixoto, J. M., Santos, S. M. E., Faria, R. M. D. de, \& Moura, A. S. (2018). Processos de Desenvolvimento do Raciocínio Clínico em Estudantes de Medicina. Revista Brasileira de Educação Médica, 42(1), 75-83.

Penteado, R., \& Costa, B. (2021). Trabalho Docente com Videoaulas em EAD: Dificuldades de Professores e Desafios para a Formação e a Profissão Docente. Educação Em Revista, 37. https://doi.org/10.1590/0102-4698236284

Rosner, E. R., Reiss, E., Warren, N. G., Shadomy, H. J., \& Lipman, H. B. (2002). Evaluation of the status of laboratory practices and the need for continuing education in medical mycology. American Journal of Clinical Pathology, 118(2), 278-286. https://doi.org/10.1309/RR95-P7DN-41LW-1LGQ

Rothan, H. A., \& Byrareddy, S. N. (2020). The epidemiology and pathogenesis of coronavirus disease (COVID-19) outbreak. Journal of Autoimmunity, 109, 102433. https://doi.org/https://doi.org/10.1016/j.jaut.2020.102433

Sohrabi, C., Alsafi, Z., O’Neill, N., Khan, M., Kerwan, A., Al-Jabir, A., Iosifidis, C., \& Agha, R. (2020). World Health Organization declares global emergency: A review of the 2019 novel coronavirus (COVID-19). International Journal of Surgery, 76, 71-76, https://doi.org/https://doi.org/10.1016/j.ijsu.2020.02.034

Spielman, A. I., \& Sunavala-Dossabhoy, G. (2021). Pandemics and education: A historical review. Journal of Dental Education, 85(6), 741-746. https://doi.org/https://doi.org/10.1002/jdd.12615

Srinivasan, M., Wilkes, M., Stevenson, F., Nguyen, T., \& Slavin, S. (2007). Comparing Problem-Based Learning with Case-Based Learning: Effects of a Major Curricular Shift at Two Institutions. Academic Medicine, 82(1).

Steinbach, W. J., Mitchell, T. G., Schell, W. A., Espinel-Ingroff, A., Coico, R. F., Walsh, T. J., \& Perfect, J. R. (2003). Status of medical mycology education. Medical Mycology, 41(6), 457-467. https://doi.org/10.1080/13693780310001631322

Vallabhaneni, S., Mody, R. K., Walker, T., \& Chiller, T. (2016). The Global Burden of Fungal Diseases. Infectious Disease Clinics of North America, 30(1), 1-11. https://doi.org/https://doi.org/10.1016/j.idc.2015.10.004

Vermelho, A.B, Pereira, A.F., Coelho, R.R.R., Souto-Padrón, T. C. B. S. (2014). Práticas de microbiologia. Guanabara Koogan.

Vieira, J., Vieira, M., \& Pasqualli, R. (2017). Estudo de caso como estratégia de ensino para a Educação Profissional e Tecnológica. Série-Estudos - Periódico Do Programa de Pós-Graduação Em Educação Da UCDB, 22, 143. https://doi.org/10.20435/serie-estudos.v22i44.1012 